\title{
Aptitude à la marche de vaches laitières de types génétiques différents : influence d'un exercice répété sur la production laitière
}

\author{
JB Coulon 1, JP Garel 2 \\ avec la collaboration technique d'E Albaret et JC Bonnefoy \\ 1 Laboratoire adaptation des herbivores aux milieux, Inra, 63122 Saint-Genès-Champanelle; \\ 2 Inra, domaine expérimental de la Borie, 15190 Marcenat, France
}

(Reçu le 17 août 1995 ; accepté le 21 novembre 1995)

\begin{abstract}
Résumé - L'effet d'une marche répétée sur la production et la composition du lait a été étudié au pâturage sur 60 vaches laitières multipares en pleine lactation de race Holstein, Montbéliarde et Tarentaise. Intrarace, trois lots de six à huit vaches ont été constitués. Le premier lot (lot $M$ ) a marché $9,6 \mathrm{~km} / \mathrm{jour}$ tous les 2 jours pendant 9 jours. Le second lot (lot T) restait sur une aire d'attente pendant la durée de la marche du lot $\mathrm{M}$. Le troisième lot (lot $\mathrm{P}$ ) restait au pâturage pendant les marches. En moyenne sur les 9 jours, la production laitière a été significativement plus faible dans les lots $\mathrm{M}$ que dans les lots $T$. L'écart a été de $0,7 \mathrm{~kg} / \mathrm{jou}$ chez les vaches Tarentaises et de $1,5 \mathrm{~kg} / \mathrm{jour}$ chez les vaches Holstein et Montbéliardes. La répétition de la marche n'a entraîné ni réduction ni accentuation de l'effet sur la production. Quelle que soit la race, il n'y a pas eu de différence significative entre les lots $P$ et $T$. Au cours des 6 heures suivant la marche, les vaches des lots $M$ sont restées couchées plus longtemps (+ 33 minutes, $p<0,01)$ et ont passé moins de temps à pâturer $(-29$ minutes, $p<0,01)$ que celles des lots $T$.
\end{abstract}

vache laitière / aptitude à la marche / production laitière

Summary - Effects of repeated walking on dairy cow performances. The effect of repeated walking (9.6 km/day every 2 days during 9 days) on milk production and composition was analysed on 60 multiparous dairy cows of three different breeds (Holstein, Montbéliarde and Tarentaise) at pasture. Performances of walking cows (group $M$ ) were compared to those of cows that remained during walking of the $M$ group in a dry lot with neither water nor food (group $T$ ), and to those of cows that remained grazing (group P). On average on the 9 day period of walking, milk yield was significantly lower in the $M$ than in the $T$ group. The difference was lower in the Tarentaise cows $(0.7 \mathrm{~kg} / \mathrm{day})$ than in the Holstein and Montbeliarde cows $(1.5 \mathrm{~kg} /$ day). The immediate effect of walking was the same during the five walkings. Whatever the breed, there was no significant differences between $P$ and $T$ groups. During the 6 hours following the walking, $M$ cows remained lying longer ( $+33 \mathrm{~min}, \mathrm{P}<0.01$ ) and spent less time grazing $(-29 \mathrm{~min}, \mathrm{P}<0.01)$ than the $T$ cows. 


\section{INTRODUCTION}

Dans certaines conditions de milieu (pâturage peu abondant ou accidenté, alpages), le déplacement représente une activité importante pour les animaux, et ses conséquences sur l'augmentation de leurs besoins et leurs performances de production peuvent ne pas être négligeables (Ribeiro et al, 1977 ; Lawrence et Stibbards, 1990 ; Matthewman et al, 1993). Dans ces conditions, les distances parcourues peuvent varier selon le type d'animal (Arnold et Dudzinski, 1978 ; Lathrop et al, 1988), mais peu de travaux ont été réalisés avec des vaches laitières (Lamb et al, 1981; Matthewman et al, 1989). Dans des essais précédents (D'Hour et al, 1994), nous avons montré que des vaches Tarentaises se déplaçaient plus rapidement et plus facilement que des vaches Holstein et Montbéliardes, et que la diminution de production consécutive à une marche prolongée ( 9 à $12 \mathrm{~km}$ ) était moins accentuée. Ces résultats ont été obtenus suite à un exercice unique. L'objectif de cette étude est d'analyser l'effet d'une marche répétée sur la production laitière, selon la race des vaches, de manière à préciser si les animaux s'habituent à un exercice répété ou au contraire y sont de plus en plus sensibles. On cherchera par ailleurs à séparer l'effet propre de l'effort physique (animaux soumis à une marche) de celui de la réduction du temps de pâturage (animaux privés de pâturage durant la marche de leurs congénères).

\section{MATÉRIEL ET MÉTHODES}

\section{Animaux et traitements expérimentaux}

Soixante vaches multipares de race Holstein (19), Montbéliarde (19) et Tarentaise (22), ayant vêlé entre le 10 novembre et le 28 février (14 décembre en moyenne) ont été utilisées dans un essai estival de 4 semaines ( 6 juillet au 3 août). Trois lots de six à huit vaches par race ont été constitués sur la base du numéro de lactation, du poids vif et de la production laitière au cours des deux dernières semaines du mois de juin. Toutes ces vaches étaient indemnes de boiteries avant l'essai. Depuis 2 mois, elles avaient l'habitude de marcher environ $3 \mathrm{~km}$ par jour pour rentrer à l'étable matin et soir lors de la traite. En plus de cette marche habituelle, le premier lot (lot $M$ ) a réalisé une marche expérimentale tous les 2 jours entre le 16 et le 24 juillet (soit cinq marches). Le second lot (lot $\mathrm{T}$ ) restait sur une aire d'attente pendant la durée de la marche du lot $M$, sans possibilité de manger ou de boire. La surface de l'aire d'attente permettait théoriquement à ces animaux de se coucher. En pratique, ils sont restés debout durant tout le temps de la marche du lot $M$. Le $3^{e}$ lot (lot $P$ ) restait au pâturage durant les marches.

Les marches ont été réalisées le matin après la traite (entre $9 \mathrm{~h}$ et $11 \mathrm{~h} \mathrm{30}$ ), sur un circuit de $3,2 \mathrm{~km}$ présentant un dénivelé de $80 \mathrm{~m}$, parcouru trois fois consécutivement. Les caractéristiques de ce parcours et le mode de conduite des animaux durant la marche ont été décrits en détail par ailleurs (D'Hour et al, 1994). Les animaux n'avaient pas la possibilité de manger ou de boire durant la marche. Les trois lots $M$ de chaque race étaient conduits ensemble pendant la marche. À la fin de chaque exercice, les animaux des lots $M$ et $T$ rejoignaient ceux des lots $P$ dans la parcelle pâturée.

Au cours de l'essai, tous les animaux ont été conduits ensemble sur deux parcelles de prairie naturelle de respectivement 10,8 et 6,2 ha, exploitées en pâturage libre. La première parcelle a été utilisée entre le 6 et le 15 juillet et après le 24 juillet, la seconde a été utilisée pendant la période de marche (16 au 24 juillet). La valeur nutritive de l'herbe à l'entrée dans chacune des deux parcelles, estimée d'après les tables Inra, a été en moyenne de 0,9 UFL, $131 \mathrm{~g}$ PDIN et 101 $\mathrm{g}$ PDIE $/ \mathrm{kg}$ MS. Les vaches qui produisaient en début d'essai plus de $20 \mathrm{~kg}$ de lait par jour (soit 14 vaches Holstein et 3 vaches Montbéliardes) ont reçu de l'aliment concentré (offert avant la traite), à raison de $1 \mathrm{~kg}$ par tranche de $2,5 \mathrm{~kg}$ de lait audessus de cette production. En moyenne, la quantité de concentré distribué a été de respectivement 1,$0 ; 0,1$ et $0,0 \mathrm{~kg} / \mathrm{jour}$ pour les vaches Holstein, Montbéliardes et Tarentaises. 


\section{Mesures}

La production laitière a été pesée individuellement à toutes les traites. La composition chimique du lait (taux butyreux et protéique) a été analysée tous les jours sur chaque traite entre le 14 et le 29 juillet, et 2 jours par semaine les semaines précédentes et suivantes.

Le comportement des animaux au pâturage au cours du premier et du cinquième jour de marche a été observé, individuellement, par notation visuelle des activités (debout, couché, pâturage) toutes les 5 minutes entre le retour au pâturage et la traite du soir (soit durant respectivement 3 heures 40 minutes et 3 heures 50 minutes les jours 1 et 5) pour les lots $M$ et $T$, et entre la traite du matin et celle du soir pour les lots $P$ (soit durant respectivement 6 heures et 5 heures 50 minutes les jours 1 et 5 ).

\section{Analyses des données}

La production et la composition du lait moyenne entre la première traite suivant la première marche et la troisième traite suivant la dernière marche (soit 18 traites) ont été calculées pour chaque vache. Ces données ont été traitées par analyse de variance (procédure GLM, Sas 1987) en introduisant dans le modèle le traitement, la race, l'interaction traitement $x$ race, ainsi que la valeur de la variable analysée observée la semaine précédant la première marche (covariable). Les interactions entre la covariable et le traitement, n'ayant jamais été significatives, n'ont pas été conservées dans le modèle final. Pour les variables de comportement, le modèle a été le même ; la covariable introduite a été la production laitière la semaine précédant la première marche. Les données présentées au tableau I sont celles ajustées par ce modèle. L'ajustement à la covariable a été réalisé intrarace.

Dans le lot M, l'effet instantané de la marche sur la production laitière a été mesuré en calculant pour chaque vache et pour chaque marche l'écart entre les traites précédant et suivant la marche. Ces données ont été traitées par analyse de variance en mesures répétées (Sas, 1987) en introduisant dans le modèle la race et la production laitière la semaine précédant la première marche.

\section{RÉSULTATS}

Au cours de la période de marche, la production laitière a été en moyenne plus faible dans les lots $M$ que dans les lots $P$ et $T$ (de respectivement 1,2 et $0,8 \mathrm{~kg} / \mathrm{jour}, p<0,01$, tableau 1). L'écart entre les lots $M$ et $T$ a été deux fois plus faible chez les vaches Tarentaises $(-0,7 \mathrm{~kg} / \mathrm{jour})$ que chez les vaches Holstein et Montbéliardes $(-1,5 \mathrm{~kg} / \mathrm{jour})$. Cette interaction n'est cependant pas significative. Cette diminution de la production laitière s'est accompagnée d'une augmentation significative du taux butyreux $(+1,6 \mathrm{~g} / \mathrm{kg}$ entre les lots $M$ et $T+P, p<0,05)$, de sorte que les quantités de matières grasses produites ont été en moyenne peu différentes d'un lot à l'autre. Le taux protéique n'a pas été significativement différent d'un lot à l'autre, et les quantités de matières protéiques produites ont donc été inférieures de 23,40 et $50 \mathrm{~g} / \mathrm{jour}$ chez les Tarentaises, les Montbéliardes et les Holstein, soit un écart de 7 à $11 \%$.

Quelle que soit la race, aucun des écarts observés entre les performances des vaches des lots $P$ et celles du lot T n'a été significatif. Les vaches Montbéliardes et Holstein du lot $\mathrm{P}$ ont cependant produit en moyenne un peu plus de lait que celles des lots T.

En moyenne, l'effet instantané de la marche n'a pas été significativement différent d'un jour à l'autre. II a cependant été plus faible le dernier jour $(-1,1 \mathrm{~kg} / \mathrm{jour})$ que les autres $(-1,8$ à $-2,1 \mathrm{~kg} / \mathrm{jour})$. Chez les vaches Holstein et Montbéliardes, la production laitière a chuté fortement lors des deux traites suivant la marche et est remontée au niveau du lot $P$ dès les troisième et quatrième traites. Cette évolution a été beaucoup moins marquée chez les vaches Tarentaises (fig 1), de sorte que l'effet instantané de la marche a toujours été plus faible $(p<0,01)$ chez les vaches Taren- 


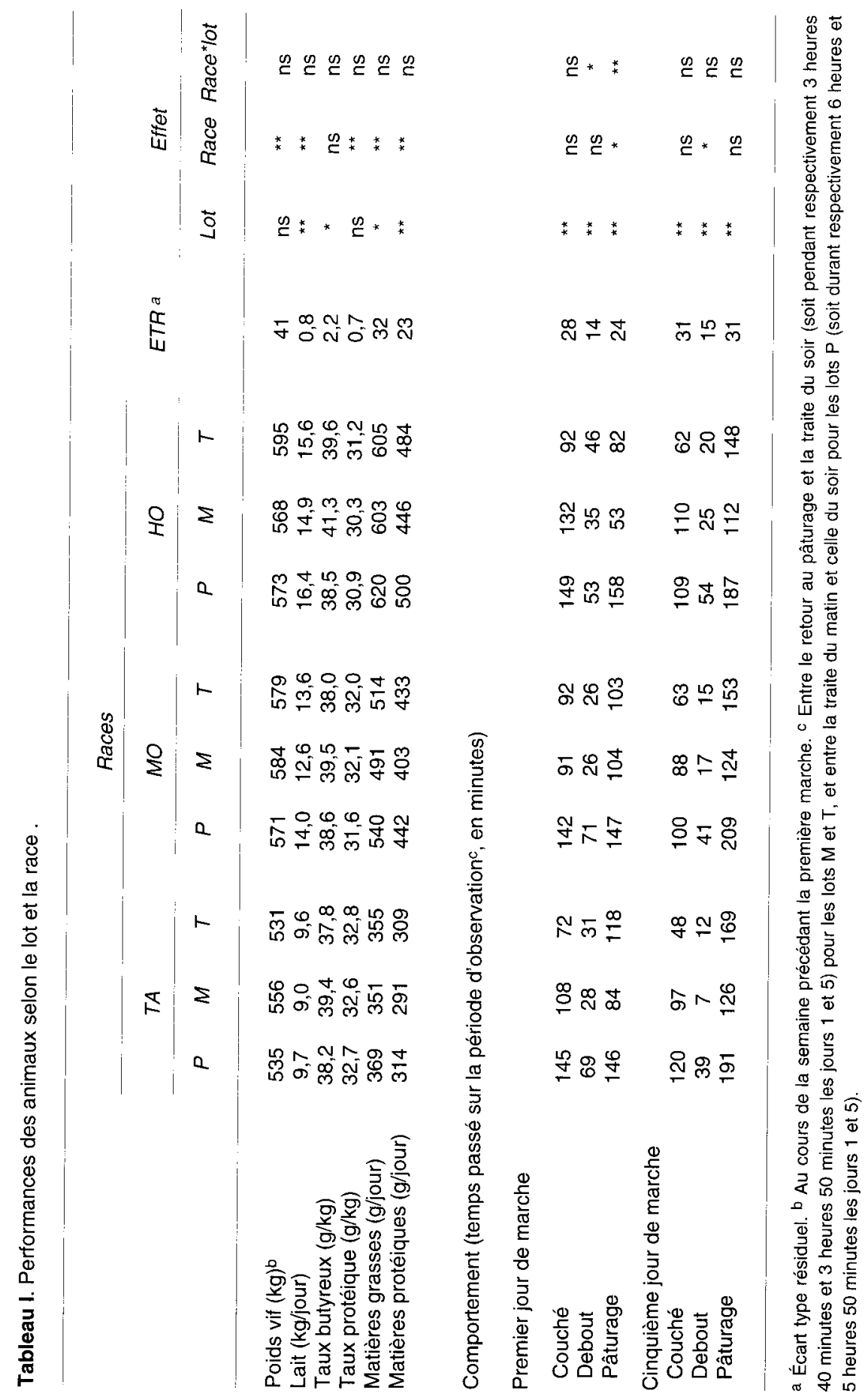


taises $(-0,5$ à $-1,1 \mathrm{~kg} / \mathrm{jour}$ selon les jours) que chez les vaches Holstein et Montbéliardes $(-1,3$ à $-2,7 \mathrm{~kg} /$ jour selon les jours). Intrarace cet écart a été indépendant du niveau de production des animaux. Le taux butyreux, et dans une moindre mesure le taux protéique, a présenté une évolution inverse. Les vaches du lot $T$ ont aussi présenté une diminution de la production le jour suivant la marche du lot $M$, mais moins importante et variable d'un jour à l'autre (pratiquement nulle le premier et le cinquième jour de marche, importante le quatrième jour).

Au cours de la semaine suivant la dernière marche, les performances des vaches des trois lots ont été voisines (fig 1).
Quels que soient la race et le lot, les vaches ont pâturé plus longtemps le cinquième jour de marche que le premier jour $(+15 \%)$. En moyenne sur les deux jours d'observation, entre la fin de la marche et la traite du soir, les vaches ayant marché sont restées plus longtemps couchées que celles maintenues dans le parc d'attente $(+33$ minutes, $p<0,01)$ et ont passé moins de temps à pâturer $(-29$ minutes, $p<0,01)$. Le premier jour de marche, cette différence n'a pas été observée chez les vaches Montbéliardes. En moyenne, les vaches Holstein des traitements $M$ et $T$ ont passé moins de temps à pâturer que les vaches Tarentaises et Montbéliardes. Cet écart est particulièrement important dans le lot $\mathrm{M}$, le premier jour de marche (respectivement 84,104 et
Fig 1. Évolution des performances des animaux au cours de l'essai (Holstein et Montbéliardes : symboles vides; Tarentaises: symboles pleins) (lot $\mathrm{T} \Delta, \boldsymbol{\Delta} ; \operatorname{lot} \mathrm{M}$ $0, \bullet ; \operatorname{lot} P \square, \mathbf{0})$.

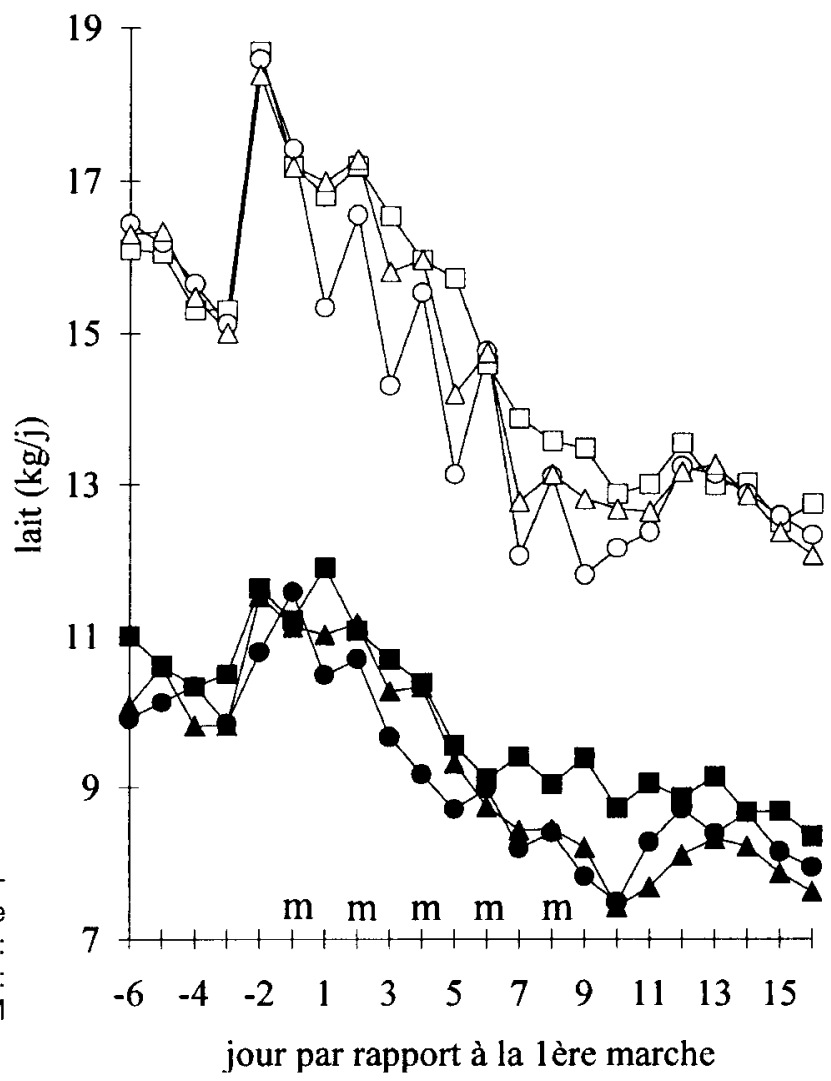


53 minutes pour les vaches Tarentaises, Montbéliardes et Holstein, $p<0,01$ ). Les vaches restées au pâturage ont au total passé respectivement 44 et 72 minutes de plus à ingérer que celles des lots $M$ et $T$.

\section{DISCUSSION ET CONCLUSION}

Cet essai montre que chez des animaux habitués à une marche modérée, une marche prolongée conduit à une diminution importante, mais de courte durée de la production laitière. II confirme en cela les premières observations réalisées par D'Hour et al (1994). Les variations concomitantes du taux butyreux sont certainement dues à un effet de concentration des matières grasses, comme c'est souvent observé lors de modifications brutales des apports alimentaires (Stobbs et Brett, 1974). L'animal mobilise ses lipides corporels pour synthétiser des matières grasses du lait qui se trouvent concentrées dans un moindre volume. Quel que soit le type de vache, la répétition de cet effort ne semble entraîner ni réduction ni accentuation significative des effets sur la production.

La diminution de production laitière est due à la fois à la limitation du temps de pâturage potentiel, comme le montre l'écart entre les lots $T$ et $P$, mais aussi à l'effort réalisé (écart entre les lots $M$ et $T$ ). Cet effort a un effet direct sur l'augmentation des besoins que l'on peut estimer, d'après les données de Ribiero et al (1977) et compte tenu des caractéristiques du parcours, à environ 3400 kcal d'énergie métabolisable, soit 1,2 UFL, si l'on retient un rendement d'utilisation de l'énergie métabolisable en énergie nette de 0,6 . Ceci correspond à $2,7 \mathrm{~kg}$ de lait, si l'on considère un rendement marginal maximal de $2,3 \mathrm{~kg} / \mathrm{UFL}$, mais vraisemblablement beaucoup moins compte tenu du rendement marginal de l'énergie habituellement observé autour de la couverture des besoins $(0,7 \mathrm{~kg} / \mathrm{UFL}$, Coulon et Rémond, 1991). Une partie importante de l'effet de la marche est donc un effet indirect, dû à la fatigue des animaux : ceci se traduit par une diminution du temps d'ingestion au profit du temps couché dans les heures qui suivent la marche. Ce phénomène a été particulièrement net chez les vaches Holstein. La part respective de ces deux effets semble variable, comme le montre la variabilité de l'écart de production entre les lots $T$ et $P$ d'un jour à l'autre. Cette variabilité est vraisemblablement liée aux conditions de pâturage. Les 2 jours où nous avons observé le comportement des animaux, les vaches des lots $T$ ont passé beaucoup plus de temps à ingérer que celles des lots $M$, ce qui explique que leur production n'ait été que peu affectée. Il est possible que les autres jours les conditions de pâturage aient été moins favorables et n'aient pas permis un rattrapage suffisant des vaches des lots $T$. Pourtant, les conditions climatiques ont été peu variables au cours de l'essai : la température moyenne a été comprise entre 15,7 (quatrième jour de marche) et $21,2^{\circ} \mathrm{C}$ (troisième jour de marche), et il n'a plu qu'un jour (entre le troisième et le quatrième jour de marche).

Comme nous l'avions déjà observé (D'Hour et al, 1994), la diminution de la production laitière consécutive à la marche a été plus marquée chez les vaches Holstein et Montbéliardes que chez les vaches Tarentaises. Cet écart est vraisemblablement dû d'abord au niveau de production différent des animaux qui conduit les vaches Tarentaises à être en bilan énergétique plus excédentaire que les vaches Holstein et Montbéliardes et donc moins sensibles à une augmentation des besoins. Compte tenu des caractéristiques moyennes de l'herbe disponible, l'écart entre ces 2 types d'animaux peut être estimé à environ 1,5 UFL (Coulon et Faverdin, 1994). Ceci explique que les vaches Tarentaises soient moins sensibles à l'augmentation des besoins énergétiques liée à la marche. 
Cependant, indépendamment de cet effet du bilan énergétique, il est aussi possible que les vaches Tarentaises présentent une capacité à mieux tamponner les variations brutales des conditions de milieu, comme cela a été mis en évidence au cours de variations importantes de températures (Colleau et al, 1979).

\section{RÉFÉRENCES}

Arnold GW, Dudzinski ML (1978) Ethology of Free-Ranging Domestic Animals. Elsevier, Amsterdam, the Netherlands

Colleau JJ, Singh M, Dupont M, Tanguy D, Briend $M$, Chambeyron JJ, Bibé B (1979) Analysis of the genetic variation for adaptation to a short thermal stress on young Bos taurus cattle. Ann Génét Sél Anim 11, 187-221

Coulon JB, Rémond B (1991) Réponses de la production et de la composition du lait de vache aux variations d'apports nutritifs. INRA Prod Anim 4, 49-56

Coulon JB, Faverdin $\mathrm{P}$ (1994) Vaches laitières. In : INRAtion, logiciel de calcul et d'analyse de rations pour les ruminants, version 2.5 (J Agabriel, P Champciaux, C Espinasse, eds), Cnerta, Dijon
D'hour P, Hauwuy A, Coulon JB, Garel JP (1994) Walking and dairy cattle performance. Ann Zootech 43, 369-378

Lathrop WJ, Kress DD, Havstad KM, Doornbos DE, Ayers EL (1988) Grazing behaviour of rangeland beef cows differing in milk production. App/ Anim Behav Sci 21,315-327

Lawrence PR, Stibbards RJ (1990) The energy cost of walking, carrying and pulling loads on flat surfaces by brahman cattle and swamp buffalo. Anim Prod 50, 29-39

Lamb RC, Anderson MJ, Walters JL (1981) Forced walking prepartum for dairy cows of different ages. J Dairy Sci 64, 2017-2024

Matthewman RW, Merrit J, Smith AJ, Phillips P, Oldham JD (1989) Effects of exercise on lactational performance in cattle. Proc Nutr Soc 92A

Matthewman RW, Oldham JD, Horgan GW (1993) A note on the effect of sustained exercise on straw intake in lactating cattle. Anim Prod 57, 491-494

Ribiero JM, Brockway JM, Webster AJF (1977) A note on the energy cost of walking in cattle. Anim Prod 25, 107-110

Sas (1987) SAS User's Guide: Statistics. Sas Institute Inc, Cary, NC, États-Unis

Stobbs TH, Brett DJ (1974) Milk yield and composition of milk and blood as indicators of energy intake by Jersey cows. Aust J Agric Res 25, 657-666 